\title{
Model to Improve the Assimilation of Contents in Programming
}

\author{
Ivelisse Teresa Machín Torres ${ }^{1}$ \\ Professor, Technical Department, José Martí University, Sancti -Spiritus, Cuba ${ }^{1}$
}

\begin{abstract}
The contemporary world requires each day more intensity in the use of technologies, this requires an improvement in the programming teaching. This work provides a model that contributes to the implementation of concepts and methods of Object Oriented Programming using multiple intelligence, in such a way that it favors the knowledge management and improve the orientation to the solution of problems in the informatics professionals. An Intelligent Tutor System component was developed as part of the model, based on an integration of Artificial Intelligence techniques, taking into account multiple intelligences. The component intelligent tutor system, contributes to the traditional architecture of tutor systems. An administrator interface allows professor the management of the data referred to the activities developed by the students in the system, thus the analytical extraction and the forecast capacity of the students through data mining techniques. The model was tested under validation and favorable outcomes were obtained.
\end{abstract}

Keywords: Learning, multiple intelligence, knowledge management, model, intelligent tutor system.

\section{INTRODUCTION}

The use of information technologies and its better application for them is currently a key issue in the development of any nation. The economic, social and cultural profits obtained from the technological factor will belong to those who manipulate them properly [1].

The use of virtual spaces for technical and professional training has generated new applicable forms to the teaching-learning process, in which face-to-face sessions are minimized. These new forms provide more speed and efficiency in the communication process, as well as access to a wider range of information sources than those provided through traditional media. By increasing the technological factors, the flexibility in the integration of techniques in terms of space, time, content offer, didactic resources, grows and also it improves the access to educational systems [2].

The profitability and range of this training is possible due to an increase of the specialist's areas with better computer and logistical services, modern infrastructures and an easy creation and implementation of programs. The breaking of physical gaps is possible by using internet as the main channel to the process of knowledge management, breaking with the presence.

There are active agents that modify and use the methods and concepts of object-oriented programming (OOP). The OOP is a programming paradigm that appears to innovate the way to obtain results. The objects manipulate input data to obtain specific output data, where each object provides special functionality.

Many of pre-designed objects of the current programming languages allow grouping in libraries or libraries, 
Studies describing the successful application of ITS in different areas of knowledge are reported [6]. Their application brings significant gains in learning according to other types of systems used to support the teachinglearning process [7]. The main cause of these positive results is the feedback the student receive during the activities [8]. It is emphasized that there is a direct relationship between the amount of feedback and these gains [9].

Within the ITS are systems such as Lisp Tutor [10], ELMART [11], SQLT-Web [12], and HESEI [13]. These systems show that their application favors the achievement of learning gains. In contrast to this effectiveness, only SQLT-Web has been used exhaustively in real-world contexts [14], and the rest only in laboratory tests with students in conditions that can be considered as controlled [15].

In the consulted researches, the following difficulties are noticed:

1. The specific intelligences of each student are not identified and it is not given an appropriate treatment in the work sessions.

2. It is not significant to find architectures that favor reusable components among systems, even within the same area of knowledge [16]. This causes that ITS development has to be carried out from the initial point, prolonging the delivery times and increasing production costs [17].

3. Inclusion of new activities in time of exploitation is not considered [18]. These are added during the development, as part of the tasks to be done at that stage, conceiving the systems in a form of black boxes where users employ the knowledge added during the development.

4. In relation to the above, the capacity of teachers is limited to extend and modify knowledge according to their considerations and to the specificities of their students. For its extension is necessary to produce new versions, which usually occurs at a slower speed than the needed by end users.

5. The encoded knowledge, with no evolution, causes that the obsolescence process of the system to be accelerated.

A previous study carried out in computer-related careers groups at José Martí University in the province of SanctiSpíritus, Cuba [19] revealed some essential deficiencies and limitations that are presented in the OOP topics, Including the following:

1. It is not always possible to identify the classes correctly. 2. Inheritance and Polymorphism are frequently misused.
3. Sometimes are used some methods that do not clearly express what it is needed.

4. Very often there is a lack of objectivity in the management of Encapsulation.

5. In the handling of Language Semantics, repeated difficulties are presented.

6. It can be affirmed that the inability to identify the essence of a problem is often observed.

The practice shows that often the required supports do not exist and it is necessary to use techniques that, in some cases, are ineffective; due to they do not take as a base the relations student-professor-contents, which is indispensable for the correct appropriation of knowledge. This has as a result the lack of motivation and a certain rejection to the content for considering them difficult to learn.

According to the non-consideration of the intelligence of each student and the adaptation of the ITS to those characteristics, it is convenient to analyze that the role of the programming requires a combination of intelligences and the capacity to develop them, since they are indispensable competences such as to create algorithms through the pseudolanguage technique, manage basic structures, it means selection, iteration, assignment, represent problems and real data through classes and objects, respect style rules, debug programs. Psychological theories about intelligence have recognized the existence of various types of intelligence, understanding that it is not a simple category, but a multidimensional one.

The theory of multiple intelligences proposed and enriched by various authors [20], breaks the traditional paradigm of intelligence as unique and general and indicates its condition of plurality. This new vision of intelligence allows to recover the human condition in relation to its multiple capacities of cognition and it generates new pedagogical and institutional practices in education. In this theoretical framework is made emphasis on the value of the cultural context for the development of the human intellect. Based on this theory it is believed that people are different and have several thinking skills and many ways of learning. Some of the types of intelligence proposed are: mathematical logical intelligence, verbal intelligence, interpersonal intelligence, intrapersonal intelligence, musical intelligence, naturalistic intelligence, body kinesthetic intelligence, visual space intelligence and digital intelligence.

Every individual has a certain intellectual performance based on his intelligence including the one called emotional intelligence. These intelligences are combined, cross-linked, and employed in various forms and intensities, but always in a personal and unique way. Due 
to each individual is capable of asking himself, generating and solving problems, to have the ability to make new products or solutions, have some skills and do not have some others, and to be aware of what he/she does. This involves the elements of the biological talents of the individual, of his interaction with the surrounding world and the cultural accumulation he has because of his origin and social or ethnic belonging.

To take these intelligences into account would favor the availability of supports to stimulate the enthusiasm for knowledge appropriation and the fortification of confidence to solve problems with new resources every day. The consideration of multiple intelligences for the creation of ITS can, in this way, contribute to a better development and use of these new technologies.

In spite of the general use of the OOP, its well practice is not taken into consideration. It is necessary to carry out researches to identify some of the main difficulties. The consideration of multiple intelligences is a type of study that prevails to achieve the correct model that includes an ITS to the conditions of each student, favoring a better learning process.

It is a fact the necessity of researching and finding ways that help us to face the challenge of improving and spreading the programming teaching. As well as to contribute to a better solution to the different problems concerning to education, economy, science, medicine and informatics itself. For that reason the following research problem is stated:

The students from careers with an informatics profile have some difficulties in the assimilation of contents and the solution of problems related to the OOP. To face the mentioned problem, the following hypothesis is formulated:

If there is a developed and applied model that relates concepts and methods of the OOP using the MI, the knowledge management would be favored, the appropriation of knowledge would improve and the orientation to the solution of problems related to careers from an informatics profile.

The general objective of this research is to develop a model that relates concepts and OOP methods, using multiple intelligences to favor the knowledge management, to improve the appropriation of knowledge and the orientation to the solution of problems related to careers from the informatics profile.

\section{III.METHODOLOGICAL DESIGN}

An easy way to comply with A number of scientific research methods, including analysis and synthesis, interviews and surveys, were applied to prepare the proposal.

A documentary analysis related to ITS and Object Oriented Programming was carried out, as well as the techniques used. From this analysis a system was elaborated, starting from an integration of the techniques of Artificial Intelligence, and its development at the same time with the changes in teaching methods and even in the form of conceiving learning and the education proposed by the theory of multiple intelligences. A layered architectural style was applied.

When the system was finally designed, the validation was carried out by specialist's criteria, as well as validation by users' criteria, applying surveys to the effect.

\section{IV.RESULTS AND DISCUSSION}

\section{A. Description of model}

For the purpose of the present research the Model is considered as an ideal representation of the object of study that involves those elements and relations that are considered essential and it systematizes the modeled object. It reflects the reality according to the researcher's intention.

It is defined the Model for the application of OOP using multiple intelligences as the conceptual representation that involves tools, methodologies, frameworks and an inference system to facilitate the knowledge management regarding programming. This model, using the multiple intelligences, is based on the following principles, qualities and premises.

The principles that support the proposed model for the application of programming are standardization, interoperability, flexibility, relevance, functional independence and updating.

Among the qualities of the model is the extension, the systemic approach, the integrality and the possibility of continuous improvement. In view of their application, they take into account premises such as the qualification of the developers, the support of the institution and the existence of didactics teaching materials well classified.

The main components of the model are: Smart Profile Management, Intelligent Tutor System, Technology Management and Knowledge Management.

The core of the functional model is composed by components related to each other as shown in Fig. 1. 


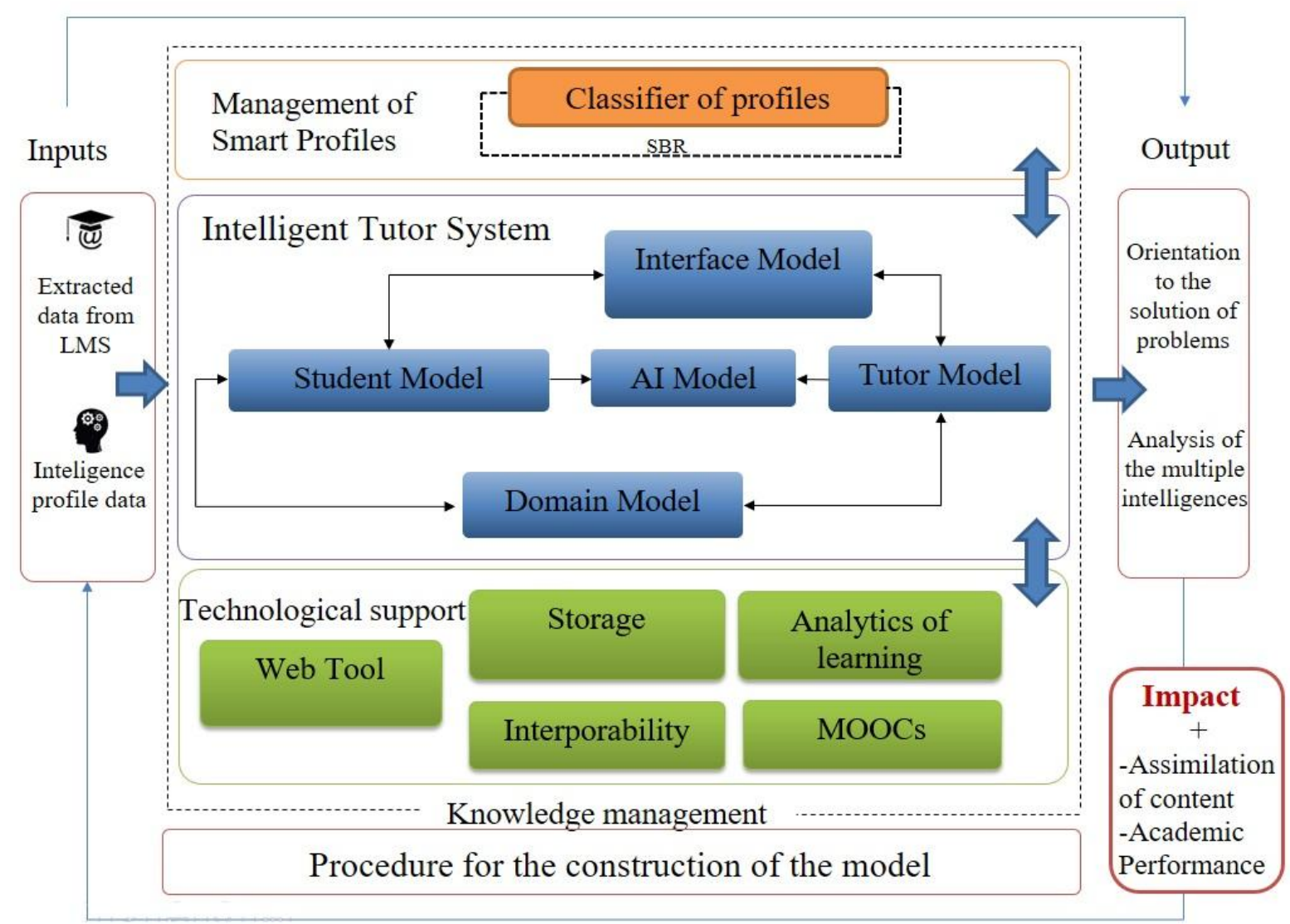

Fig. 1 General theoretical model (own elaboration).

\section{B. Description of components of the model}

The components of the model are explained in detail below.

1. Component: Management of Smart Profiles.

This component is based on a basic knowledge expressed in rules and a tool for the creation of diagnoses as support for making decisions.

The tool called Master-ProClas was used as a subsystem with a function of classification associated to Master-Pro, ITS as such, it was carried out using the developed model, demonstrating its feasibility and the advantages of the application of the model, especially the facilities it provides.

Master-ProClas is an application developed in JAVA language with a three-layer architecture (Data Layer, Business and Interface) which provides some advantages such as strengthens because of the encapsulation, the facility of maintenance, the support and flexibility, as well as high scalability.

This system has a friendly and configurable interface. It has the advantage, as shown in Figure 1, of performing a gradual classification according to the types of intelligences and pedagogical styles.

Also, Master-ProClas allows the addition of new rules in consistency with the advances made in the psychology field, for the establishment of other types of intelligence or pedagogical styles that are in the process of analysis by the international community.

To achieve intelligence classification, the Multiple Intelligence Test is filled out [20], the rules stated previously are applied, and the system will indicate the result of the inference, showing the classification.

In the case of classification by learning styles the Test is filled [21], where the rules previously defined are applied, and the system will indicate the result of the inference, showing the classification.

The fundamental value of Master-ProClas is given by its simplicity and the friendly atmosphere it offers to users. It was developed by applying the principles of the objectoriented paradigm.

\section{Component: Intelligent Tutor System}

The ITS was developed as a web application on the Java Enterprise Edition platform (Java EE) and Java as a programming language. The applied layered architectural style guarantees a logical separation among functions of different nature. This makes it easier to protect the integrity of the components against external changes, with three well defined layers: presentation, business and access to data. 
restrictions or rules established at different levels, the

The proposed system was named Master-Pro, and it integrates Artificial Intelligence techniques together with changes in teaching methods and even in the way of conceiving learning and teaching proposed by the theory of multiple intelligences. In this system is the student himself who takes control of the process, materials and resources, adapting them to their requirements and possibilities.

The Master-Pro model includes a set of components that facilitate the application of object-oriented programming contributing to the treatment of multiple intelligences in students. This ITS for the application of OOP using multiple intelligences can be used in a wide range of subjects, and it contains a set of components and relationships among them, as seen in the central area of Fig. 1.

The Student Model stores and processes information regarding the student performance during the work sessions, they directly interact with the Master-ProClas classifier, the component for the diagnostics realization to support making decisions. In addition, you get the basic updates to the learning style questionnaire and the intelligence types questionnaire, which are the basis to work on the ITS.

As part of the Artificial Intelligence Model, a hybrid subsystem is proposed that establishes the pedagogical method of adapting teaching, the appropriate contents (sequencing of learning resources or objects) and the way of presenting them (type of multimedia or collaborative resources), all with in order to plan the optimal learning path for the student based on his / her learning profile. On the other hand, they also feedback the Domain Model, indicating the preferences of the student regarding the appearance and set of tools that configure the user interface. This component is based on the Student Model, and interacts with different eLearning platforms.

On the other hand, the Interface Model is the one in charge of providing support to the direct interaction with the different roles, student, teacher or administrator. In this conception there is not breaking with the classic structure of intelligent tutors, but this new vision implies a greater flexibility and adaptation of new domains to the applications that use it.

This system, made up by several models, enhances the traditional architecture of tutor's systems, made by experts in the subject, which are related to each other and on which students can navigate. It also offers an administrative interface that allows the teacher to manage data related to the activities developed by the students in the system, as well as the extraction of analytics and prediction of school performance.

The main inputs of the general theoretical model are those people turned into users, computerized processes, contents that needs to be treated and the methodological indications that are part of the context where the components of the ITS will be deployed.

The general outputs of the general theoretical model are reflected in the configurations, reports or analyzes based on event logs, user identities, established policies and accessible resources. Additionally, each component has its own inputs and outputs that can make changes or execution of actions in the other components.

The prediction of performance is done by data mining techniques, specifically the implementation of a Bayesian network so it is used as support Weka software that offers possibilities for working with these types of networks.

To carry out the extraction of analytics and performance prediction, a series of elements stored in the system are taken into account, such as: traces and information stored in the database related to: reading documentation, performing tasks, downloading files, Test realization, activities results, test results, participation in forums and chats, communication with other students, communication with the teacher requesting help and sequencing of tasks.

\section{Component: Technology Support}

Technology Support is a fundamental process in the model. It manages both tacit and explicit knowledge necessary for the operation of Master-ProClas, Master-Pro and the technology management component, which is a fundamental process.

This component allows the communication through web services between the Master-Pro component and a Learning Management System (LMS) as well as its integration into the so-called Massive Online Open Course (MOOCs or COMA, Massive and Open Online Courses) as an evolved expression of the Open education on the internet. In this way, knowledge management is supported and it is possible to use learning analytics in terms of continuous improvement in problem solving orientation, providing a practical contribution to research through the way of integration and the facilities it offers to users of any specialty.

\section{General working of the model}

For this purpose, a database is available, the MasterProClas tool and Master-Pro that provides tutoring in the topics of object-oriented programming, the making decision and advisable elements to facilitate learning. This is based on the student's intelligence profiles.

To select the learning resources, the information provided by the Master-Pro system is taken, which is a premise of the model. Taking into consideration the composition of 
intelligence profiles, pedagogical styles, as well as the level of the initial knowledge the student has, a concordance among these elements is established that allows to obtain a preliminary route of tutoring. If the route coincides with that taken by other students, then it will be necessary to provide the information in such a way that the previous performances has to be taken into account.

The knowledge management is an essential process in the model. It is managed tacit and explicit knowledge, necessary to Master-ProClas, Master-Pro and the technological management component working.

Knowledge management refers to the set of processes developed by a tutor to create, organize, store and transmit knowledge. This concept is widely used in tutor's systems that focus on the transmission of knowledge and experiences of experts in a way that is available and can be used by other students. This process involves the use of various techniques to capture, organize and store the knowledge of experts to transform it into an intellectual asset that provides benefits and can be shared.

In the Master-Pro model knowledge management is a pillar for the development of the processes that comprises it due to they are based on the use of knowledge for the application of programming. An essential element is the management of tacit knowledge with the aim of socializing it and turning it into explicit knowledge. An essential element is the management of tacit knowledge with the aim of socializing it and turning it into explicit knowledge.

On one hand the Master-ProClas acquires knowledge of a great variety of forms, according to the type of knowledge that is being searched. In the knowledge capture and creation phase, tacit or implicit knowledge is captured through a variety of techniques including specialists' interviews, focal groups, intelligent systems using particular patterns or search strategies, etc., the ones that have been employed in different stages of the research.

\section{Preliminary validation of the model}

The preliminary validation was carried out applying the specialists and users criteria:

\section{1- Validation of the model according to user criteria.}

Due to the current tendency to use ITS to achieve better management of knowledge, adaptability and interoperability and durability, José Martí University of Sancti-Spíritus province has developed a line of research aimed to find solutions that make possible to increase the Productivity of this type of resource, as well as the levels of uses.
To achieve this purpose, it has been convenient to collect information from the institutions that include training processes, in order to be able to estimate if the ITS developed is valid to improve the educational-process. To get that information a survey (annex 1) was applied with the objective of collecting user's criteria (students).

A sample of 27 students from the career Computer Science Engineering from different Cuban universities was taken. The observed variables were: knowledge management, orientation in problem solving, as well as motivation and selection of the pedagogical method. 25 out of the total number of surveyed students consider that the representation of the content offered by Master-Pro has made it easier for them to understand the key concepts of Object Oriented Programming 93\% of the surveyed.

On the other hand, $76 \%$ of the sample believe that it has helped to transform the specifications of a given problem into classes, methods, and objects.

21 students (78\%) assessed the influence of Master-Pro on their ability to clearly express an algorithm using the object-oriented paradigm.

The $100 \%$ of students believe that systems similar to Master-Pro would facilitate the teaching-learning process of other topics of other subjects.

Regarding the answers to question 4 a): What aspects would you like to change in the system? the most frequent and most important aspects were: the need of a voice processing module and supports that allow mobile learning.

2- Validation of the model according to specialist's criteria.

A survey designed for this purpose was applied to a sample of 27 specialist belonging to different Higher Education Institutions. We selected experts from more than seven years of experience as professionals and professors in the disciplines of Computer Programming and Artificial Intelligence, mastering the methodology of teaching programming and designing educational software. From the 27 specialists initially expected, 23 did the requirements set out in the survey, of which $85.0 \%$ had High proficiency level and $15.0 \%$ Medium, mostly had main teaching categories, $42.6 \%$ were Auxiliary, 51.0\% Senior, $25.2 \%$ are Masters and $70.2 \%$ are Doctors. The observed variables were: knowledge management, orientation in problem solving, as well as motivation and selection of the pedagogical method. The selected ones were shown in details of the proposed system, and a survey was applied with the elements to be assessed.

Regarding the way in which it is considered that this system allows to raise the levels of assimilation of the contents related to the Logical Programming, it can be

-


observed that $66.7 \%$ thinks it is very adequate and the remaining $33,3 \%$ that is adequate.

The $100 \%$ of the specialists believe there is harmony among all the resources available in the system to enable students to take ownership of the contents of the subject. It is evidenced that to offer treatment to the multiple intelligences on the part of the teacher increases the management of the knowledge, as well as the motivation by the programming subject. A correct selection of the pedagogical method allows students to have a better orientation in problem solving when programming, which will guide their subsequent performances.

\section{CONCLUSION}

The Master-Pro system built by combining ITSs and multiple intelligences promotes a structured and organized knowledge, which accelerates the learning process concerning the students.

In the proposed ITS model, each concept to be learned is enriched, according to different approaches with coaches, video tutorials, simulators, social interaction and other means that make knowledge acquisition easier.

The Master-Pro system is very easy to maintain and update, the modifications in the types of intelligences and the resources, are very simple, as well as the addition of new ones.

\section{REFERENCES}

[1] Aleven, «Rule-Based Cognitive Modeling for Intelligent Tutoring Systems, » de Advances in Intelligent Tutoring Systems, Springer-Verlag, 2010, pp. 33-62.

[2] R. Azevedo, M. Taub y N. Mudrick, «Technologies supporting self -regulated learning, » de The SAGE Enciclopedia of educational technology, M. Spector, C. Kim, T. Johnson, W. Savenye, D. Ifenthaler y G. Del Río, Edits., Thousand Oaks, CA: SAGE, 2015, pp. 731-734.

[3] R. Azevedo, «Metacognition and multimedia learning, » de Cambridge handbook of multimedia, Cambridge England, Cambridge University Press, 2014, pp. 647-672.

[4] K. Brawner, H. Holden y e. al., «Recommendations for Modern Tools to Author Tutoring Systems, » de Proceedings of the Interservice/Industry Training Simulation \& Education Conference (I/ITSEC), 2012.

[5] K. VanLehn, «"The relative effectiveness of human tutoring, intelligent tutoring systems, and other tutoring systems", ” Educational Psychologist, pp. 197-221, 2011.

[6] G. Paviotti y P. G. Rossi, «Intelligent Tutoring Systems (ITS): a European point of view, » de Intelligent Tutoring Systems: an overview, 2012, pp. 9-15.

[7] M. J. Eagle y T. Barnes, «Evaluation of Automatically Generated Hint Feedback, » de Proceedings of the International Conference on Educational Data Mining. S. K., Memphis, Tennessee, USA, 2013.

[8] M. Chi, K. VanLehn y e. al., «Do micro-level tutorial decisions matter: Applying reinforcement learning to induce pedagogical tutorial tactics, » de Proceedings of the International Conference on Intelligent Tutoring Systems, 2010.

[9] J. Dabolins y J. Grundspenkis, " "The Role of Feedback in Intelligent Tutoring System", » Applied Computer Systems 14, pp. 88-93, 2013

[10] B. J. Reiser y J. R. Anderson, «"The LISP tutor: it approaches the effectiveness of a human tutor", » de Byte, 1985, pp. 159-175.
[11] G. Weber y P. Brusilovsky, «"ELM-ART: An adaptive versatile system for Web-based instruction", » International Journal of Artificial Intelligence in Education 12, pp. 351-384, 2001.

[12] A. Mitrovic, «"An intelligent SQL tutor on the web",» International Journal of Artificial Intelligence in Education (IJAIED) , pp. 173-197, 2003.

[13] N. Martínez, « Model for designing Intelligent Teaching-Learning Systems using Case-Based Reasoning, » Doctoral Thesis, Central University of Las Villas., 2009.

[14] T. Dadic, «Intelligent Tutoring System for Learning Programming, » de Intelligent Tutoring Systems in E-Learning Environments: Design, Implementation and Evaluation, 2011, pp. 166-186.

[15] E. J. Altuna y L. Guibert, «Intelligent Tutoring Systems and their impact on teaching programming, » Memories of the XV International Congress of Informatics in Education, INFOREDU 2013, La Habana., 2013.

[16] M. Gujarathi y M. Sonawane, «Intelligent Tutoring System: A Case Study of Mobile Mentoring for Diabetes, »International Journal of Applied Information Systems, vol. 3, no 8, pp. 41-43, 2012.

[17] H. Meshref y I. Mohamed, «Intelligent Tutoring Systems: A New Proposed Structure, » de Proceedings of the International Conference on Advances in Computing, Communications and Informatics, TNadu, India, 2012.

[18] B. du Boulay y T. del Soldato, «Implementation of Motivational Tactics in Tutoring Systems: 20 years on, » International Journal of Artificial Intelligence in Education, pp. 170-182, 2015.

[19] I. T. Machín Torres, «Main difficulties presented by students in the teaching-learning process of Object Oriented Programming, » Research Report, José Martí University of Sancti-Spíritus, 2013.

[20] H. Gardner, Multiple Intelligences, Paidós, 2011.

[21] M. Felder y K. Silverman, «Learning Styles and Teaching Styles in Engineering Education, » Engr. Ed., 1988, pp. 674-681.

\section{BIOGRAPHY}

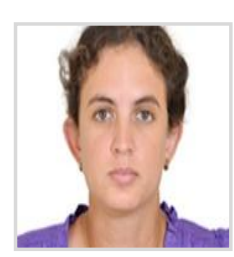

Ivelisse Teresa Machín Torres. Engineer in Computer Science, José Martí Pérez University. Researcher in the area of educational technology. Writer. Aspirant of Doctorate in Technical Sciences. 